\title{
Fiber Bragg grating dispersion-managed multisolitons
}

\author{
J. D. Ania-Castañón, P. García-Fernández, and J. M. Soto-Crespo \\ Instituto de Óptica, Consejo Superior de Investigaciones Científicas, Serrano 121, 28006, Madrid, Spain
}

Received September 7, 2000; revised manuscript received March 12, 2001

\begin{abstract}
We show that third-order dispersion, filtering, and amplification in a fiber Bragg grating dispersion-managed soliton system can lead to the formation of bound multisoliton solutions. We have found that these solutions appear due to the presence of nonsymmetrical terms in the transfer function of chirped gratings. We present an analytical approximation for the time delay of a chirped grating in the vicinity of the central frequency that takes into account higher-order dispersion terms. We also study the tolerance of these newly found multisoliton solutions to the presence of third-order dispersion in the fiber link and to random variations in the gratings parameters due to manufacture or variations in the operating conditions. (c) 2001 Optical Society of America

OCIS codes: $060.0060,060.2330,050.2770,060.5530,050.0050$.
\end{abstract}

\section{INTRODUCTION}

Dispersion-managed solitons ${ }^{1-5}$ present a number of advantages that make them very attractive for long-haul communication systems because they are now at the point of becoming a viable commercial proposition. Dispersion-managed solitons can exist when the average network dispersion is near zero ${ }^{6}$ (or even positive), thus reducing the Gordon-Haus jitter effect. ${ }^{7}$ Moreover, they are more resistant than conventional solitons to the effects of polarization mode dispersion (PMD) ${ }^{8}$ Kumar and Hasegawa ${ }^{9,10}$ introduced the concept of a quasi soliton, a chirped soliton with Gaussian tails, which appears in systems with periodic amplification and chirp reconstruction. Kumar and Hasegawa, ${ }^{9}$ and Turytsin and Mezentsev ${ }^{11}$ demonstrated that quasi solitons with strong confinement and reduced interaction between neighbors could be produced by using ideal fiber Bragg gratings (FBGs) to periodically reverse the pulse chirp. In a recent letter ${ }^{12}$ we presented a new kind of stable multipulse with a short distance between peaks that appears in nonideal fiber Bragg grating dispersion-managed soliton systems with periodic chirp reconstruction. Chirped FBGs present some interesting features as dispersioncompensating devices. They can compensate for large dispersion in a very short length. They are also compact and passive, present low loss, and act as both dispersion compensators and bandwidth filters, thus being able to limit the undesired effects of sideband generation in soliton dispersion-managed systems. ${ }^{13}$

Two conditions must be fulfilled for the formation of the bound states. First, higher-order dispersion terms in the grating-transfer function have to be big enough, and, second, certain conditions for the bandwidth ratio between the FBG and the signal must be satisfied. Given the relevance of the higher-order dispersion terms, the presence of third-order dispersion in the fiber link must have an important influence in the formation of the bound states. FBGs are sensitive to pressure, tension, and temperature variations. ${ }^{14,15}$ The external conditions of a long-haul communication system can vary greatly from point to point, thus altering the spectral response of the gratings. Also, it is impossible to manufacture two identical FBGs. Differences between the gratings in our system can have a relevant repercussion on the formation of bound states, so an analysis of the effect of small random variations of the grating parameters on the formation of multisolitons must be carried out.

We devote the present paper to these goals. We also show in some depth the evolution of a multisoliton over a single period and the mechanisms that lead to the formation of bound states. In the next section we present the theoretical model employed in our study. Section 3 is devoted to the effect of third-order dispersion on the formation of the multisolitons, and Section 4 explores the effect of the variation of the grating parameters.

\section{THEORETICAL MODEL}

For the propagation, we solve numerically the generalized nonlinear Schrödinger equation ${ }^{16}$

$$
i \frac{\partial E}{\partial z}=-\frac{i}{2} \Gamma E+\frac{1}{2} \beta_{2} \frac{\partial^{2} E}{\partial T^{2}}-\frac{i}{6} \beta_{3} \frac{\partial^{3} E}{\partial T^{3}}-\gamma|E|^{2} E,
$$

where $z$ is the distance, $E(z, T)$ is the complex slowly varying amplitude of the electric field, $\gamma$ takes account of the optical Kerr effect, $\Gamma$ is the fiber loss, $\beta_{2}$ is the secondorder dispersion parameter, and $\beta_{3}$ is the third-order dispersion parameter that we will assume to be equal to zero until the contrary is stated. The values adopted by all the parameters in our simulation are given in Table 1. We use a split-step Fourier method to simulate the propagation with a step size of $2.5 \mathrm{~km} .{ }^{16}$ The inclusion of the third-order dispersion will have an important effect on the formation of bound states as we will show in Section 3. 
The signal launched into the fiber is composed of $n$ chirped Gaussian pulses of peak power $P$, chirp $C$, and temporal width $T$, spaced $\tau_{0}$,

$E(0, T)$

$$
=P^{1 / 2} \sum_{k=0}^{n-1} \exp \left\{\left[-\frac{(1+i C)\left(T+k \tau_{0}\right)^{2}}{2 T_{0}^{2}}\right]+i \Phi_{k}\right\},
$$

where $\Phi_{k}$ is a constant phase term, specific for each pulse.

FBGs are usually modeled in dispersion-management analysis as having full reflectivity and a linear time-delay response. ${ }^{11}$ Because this is a crude approximation, and because typically $100 \mathrm{FBGs}$ are needed to periodically reverse the chirp in a $10-\mathrm{Mm}$ dispersion-managed propagation, slight deviations from this linear response may cumulatively induce some important higher-order effects. For these reasons we use a more accurate model and obtain the reflectivity and time-delay profiles for the FBGs from their coupled mode equations, which are written, in Kogelnik's notation, ${ }^{17}$ as

$$
\begin{aligned}
& \frac{\mathrm{d} R}{\mathrm{~d} z}+i \delta R=-i \kappa(z) S \exp \left[-i F\left(\frac{z}{l}\right)^{2}\right], \\
& \frac{\mathrm{d} S}{\mathrm{~d} z}-i \delta S=i \kappa(z) R \exp \left[i F\left(\frac{z}{l}\right)^{2}\right],
\end{aligned}
$$

where $R$ and $S$, respectively, are the progressive and regressive modes inside the grating, $z$ is the length coordinate, $\kappa(z)$ is the coupling coefficient that includes a Gaussian apodization function with window parameter $12, F$ is the grating chirp parameter, $l$ is the grating length, and $\delta$ is the frequency deviation with respect to the Bragg frequency at the center of the grating $(z$ $=0$ ). When these equations are numerically solved, the reflectivity is given by $|S / R|^{2}$ and the time delay by the first frequency derivative of the phase induced by the fiber grating (i.e., the phase term in $S / R$ ). The reflectivity and time delay obtained for a grating with $l=4.78 \mathrm{~cm}$, $\kappa=4.28 \mathrm{~cm}^{-1}$, and $F=300$, which compensates for the second-order dispersion suffered in the $100-\mathrm{km}$ fiber link, like the ones employed in our simulations, appear in Fig. 1.

We add random noise to the parameters of the FBGs, which take the form $P_{i}=\left\langle P_{i}\right\rangle+r_{i}\left\langle P_{i}\right\rangle$, where $i$ varies from 1 to 3 , being $P_{1}=L, P_{2}=\kappa$, and $P_{3}=F$. The $\left\langle P_{i}\right\rangle$ are the mean expected values for the parameters, and $r_{i}$ is a random factor uniformly distributed between certain negative and positive values, given by the percentage of variation that we specify in each case. We force each parameter to vary independently because all relate to different physical magnitudes, and the experi-

Table 1. Parameters of the Simulation

\begin{tabular}{lc}
\hline \multicolumn{1}{c}{ Parameter } & Value \\
\hline Nonlinear coefficient $(\bar{\gamma})$ & $2 \times 10^{-3} \mathrm{~km}^{-1} / \mathrm{mW}^{-1}$ \\
Fiber losses $(\Gamma)$ & $0.2 \mathrm{~dB} / \mathrm{km}^{-1}$ \\
Second-order dispersion coefficient $\left(\beta_{2}\right)$ & $-16 \mathrm{ps}^{2} / \mathrm{km}^{-1}$ \\
Input peak power $(P)$ & $25 \mathrm{~mW}$ \\
Initial pulse width $\left(T_{0}\right)$ & $7.8 \mathrm{ps}$ \\
Initial pulse separation $\left(\tau_{0}\right)$ & $27.34 \mathrm{ps}$ \\
\hline
\end{tabular}

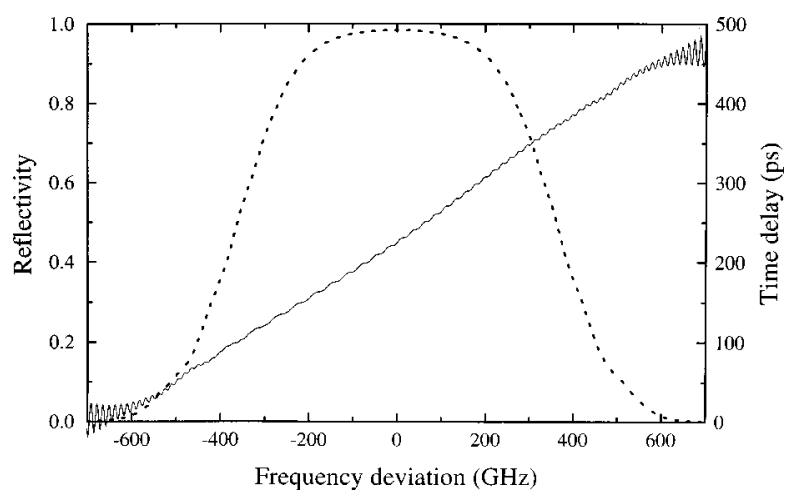

Fig. 1. Reflectivity (dashed curve) and time delay (solid curve) corresponding to one of the chirped FBGs used for periodic dispersion compensation.

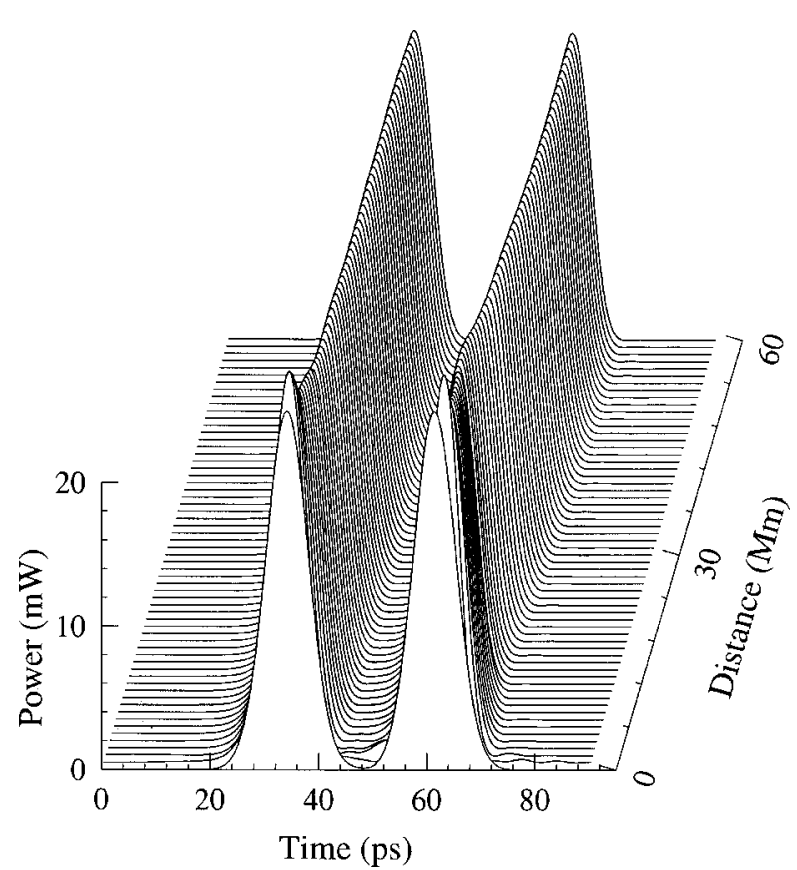

Fig. 2. Evolution of a pulse pair with initial zero phase difference over a distance of $60 \mathrm{Mm}$.

mental error in each of them is not necessarily the same. Within this approach we will be able to specify the system tolerance to the variation of any of the three characteristic parameters of linearly chirped FBGs.

Propagation takes place through distances of several megameters divided in equal cells of $100 \mathrm{~km}$. The signal is initially chirped with a chirp equal to half the chirp produced by the group-velocity dispersion in $100 \mathrm{~km}$ of propagation, but of the opposite sign, so it ideally reaches the end of the first cell with a chirp inverse to the initial one. The signal is then filtered with a linearly chirped FBG, which compensates for the second-order dispersion of the cell, and is amplified. The signal is expected to be zero chirped at the midpoint of the cell. This scheme is similar to the one proposed by Turytsin and Mezentsev ${ }^{11}$ to reduce quasi-soliton interaction and decrease the distance between neighboring pulses, though they used lowpower pulses and considered a simpler model for the gratings. Each period differs from the previous ones only in the FBG due to the random variation of its parameters. 
Afanasjev and Akhmediev ${ }^{18}$ studied the interaction between neighboring solitons in a two-dimensional phase plane that they called the interaction plane. This representation has been used by different authors for analyzing the stability and general dynamics of multisoliton solutions in different nonconservative optical systems ${ }^{19,20}$ and will be used extensively in this paper. When we use this representation, two coordinates characterize the pulse pairs in the plane: their relative phases and the

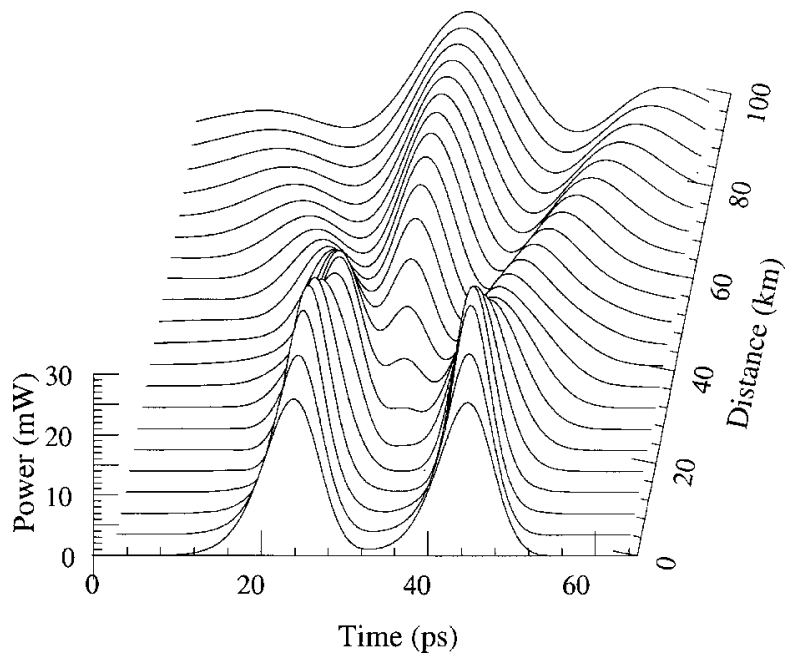

Fig. 3. Evolution of a pulse pair over a single period.
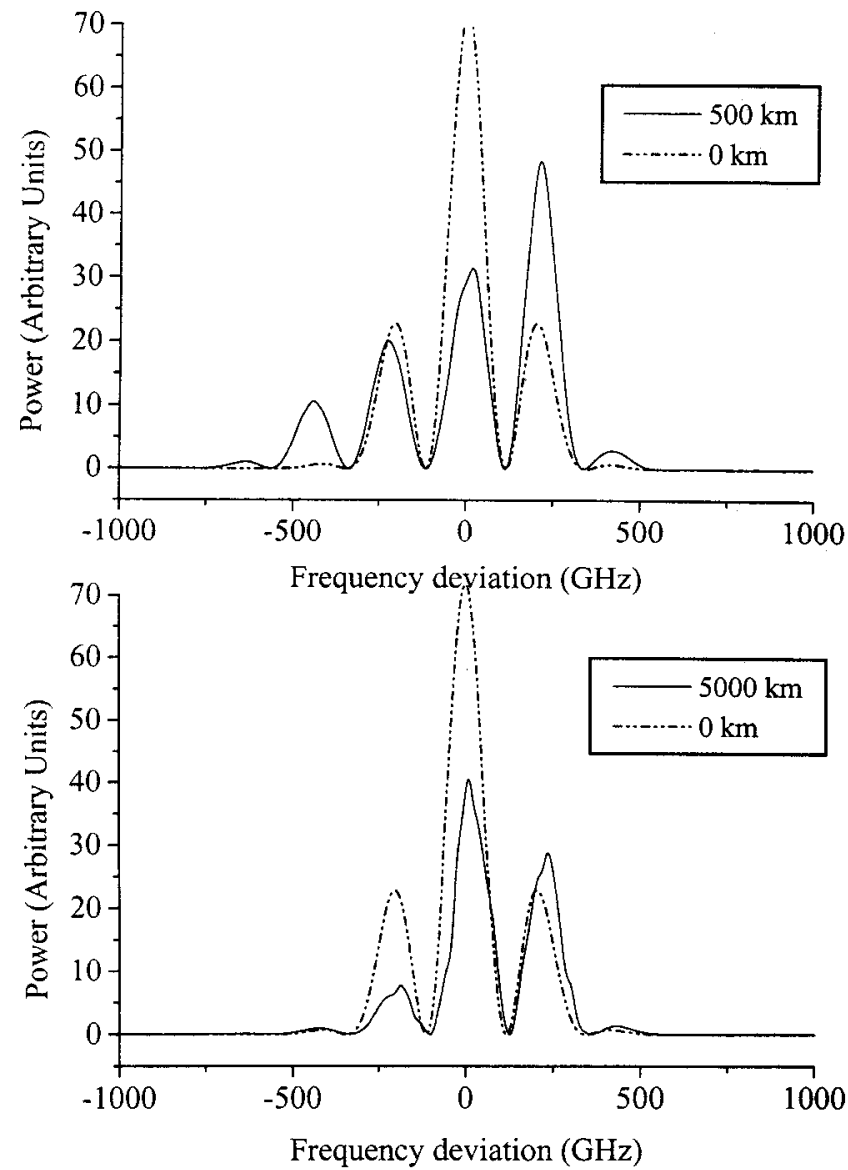

distance between peaks. When a fixed point is reached in the plane, it is indicative of the formation of a bound state.

The propagation of a pair of pulses $(n=2)$ with $P$ between 20 and $29 \mathrm{~mW}, T_{0}$ at $\sim 8 \mathrm{ps}$, and initial separations of between 2 and 5 solitons leads to the formation of a bound state of two solitons (see Fig. 2) with a separation between peaks of $\sim 29 \mathrm{ps}$ and a peak power of $\sim 25 \mathrm{~mW}$, which occurs with independence of the initial phase difference between solitons 0 and $1, \Phi_{1}-\Phi_{0}$. Bound states of three or more solitons are also possible if we impose certain initial conditions for the phase difference between solitons 0,1 , and 2 , such as $\Phi_{1}-\Phi_{0}=\Phi_{2}-\Phi_{1}$ $=\pi$, thus delaying the convergence to the bisolitonic states, because they seem to be more stable and do not combine with single pulses to form a trisoliton. ${ }^{12}$ The stability of these bound solutions is ultimately limited by the background instability that is due to the excess linear gain introduced to compensate for the losses in the gratings. Therefore we fix the input peak power of our pulses to $25 \mathrm{~mW}$, similar to power of the peaks in the bound state. This input peak power is close to the peak power of a mean soliton and is high enough to ensure an important interaction between pulses.

The evolution of the signal in a single period during the propagation through the $100-\mathrm{km}$ fiber link is depicted in Fig. 3. In order for the evolution to be adequately seen in the figure a factor that corrects for the fiber losses has been applied to the peak power of the signal. During a
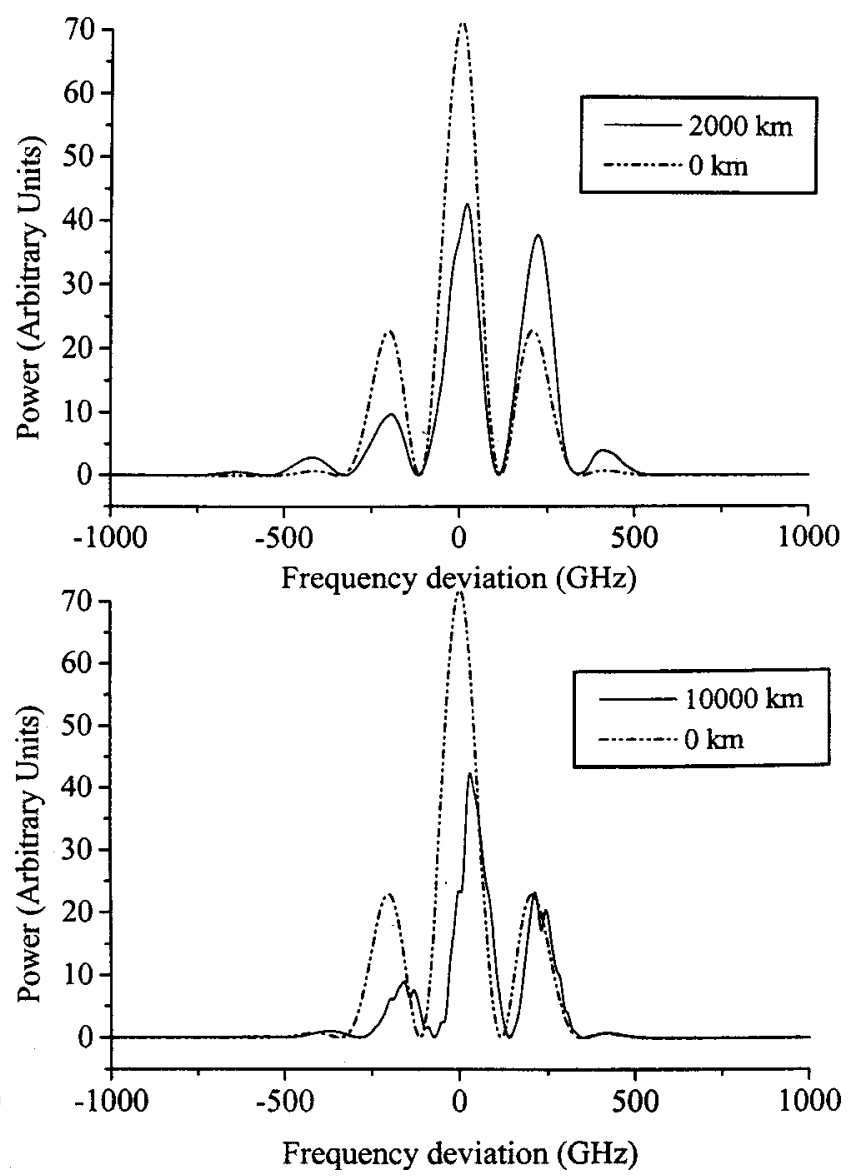

Fig. 4. Evolution of the signal frequency during propagation. 
single period the signal is affected by dispersion, selfphase modulation, and losses, and becomes wider in the spectral domain. On the other hand, pulses attract each other and nearly merge at the end of the link, and the peak power is reduced to $0.16 \mathrm{~mW}$. The signal is then reconstructed at the fiber grating, recovering its initial chirp, and is amplified. Linearly chirped gratings present low higher-order dispersion terms in their transfer function that are responsible for an asymmetry in their phase response. These effects can be strongly reduced by applying an appropriate apodization function but may reacquire influence when many gratings are used. Thus two competing effects change the signal simultaneously. The first effect is produced by the combined action of these higher-order terms, together with the nonlinear effects in the fiber link, and tends to asymmetrize the signal. ${ }^{21}$ The second is the grating filtering effect, which tends to symmetrize the signal. Both competing tendencies reach an equilibrium after a certain number of periods. Once this equilibrium is reached, the shape of the pulses is approximately fixed, in both the spectral and the time domain. In other words, only slight oscillations appear around a given point of the interaction plane. The case depicted in Fig. 2 corresponds to a pulse pair that is already oscillating close to such a stability point in the interaction plane.

The pulse asymmetrization in the frequency domain can be observed in Fig. 4. This asymmetrization is not random, but implies a global frequency displacement that occurs mainly in the first $2000 \mathrm{~km}$. After the first 5000 $\mathrm{km}$ the shape of the spectrum does not vary significantly, presenting a clear central peak and two main sidelobes, as well as a global displacement toward high frequencies. This shape is maintained in the bound state. As distance increases, some ripples appear in the spectrum that are clearly observable at $10000 \mathrm{~km}$. These ripples can be the effect on the spectrum of the noise produced by the background instability that appears due to successive amplifications and the radiation generated by the higherorder dispersion terms. ${ }^{22}$

It is important to note that two pulses initially separated a distance equal to or higher than twice the distance between peaks in the multisoliton present a very reduced interaction and do not vary their distance along the propagation.

\section{THIRD-ORDER DISPERSION}

Linearly chirped FBGs are usually considered, when one is modeling dispersion management systems, devices with linear time-delay profiles. Unfortunately real gratings present ripples in their time delay ${ }^{23,24}$ that can be reduced only through a strong apodization. Because the apodization increases the losses in the grating, it is usually necessary to find an equilibrium between the effect of the losses and the influence of the ripples.

$\mathrm{K}$. Ennser $e t a l .{ }^{25}$ used a sinusoidal model for the timedelay ripples and studied the effect of these ripples on second-order dispersion compensation. This approximation does not consider the characteristic variation of the period of the ripples with the frequency, but considers that the phase-tranfer function of the grating is symmetric, and does not introduce dispersive terms of odd order.

A more accurate approximation can be reached if we consider a linear variation for the period of the ripples with the frequency deviation (see Fig. 5),

$$
\tau_{ \pm}(\omega)=a_{1} \omega+a_{2}+b \sin \left[\frac{2 \pi}{\mp \Omega_{F} p_{0}}\left(\omega \mp \Omega_{F}\right) \omega\right],
$$

where $p_{0}$ is the period of the ripples at $\omega=0$ and $\Omega_{F}$ is the effective spectral width of the grating as a filter. The sign \pm refers to the two possible directions of increase for the period variation. In the positive case $\left(\tau_{+}\right)$, the period increases with the frequency deviation $\omega$, whereas in the negative case $\left(\tau_{-}\right)$it decreases with $\omega$. Under this approximation, changing the orientation of the FBG corresponds to changing the sign of $a_{1}$, and switching between the cases $(+)$ and $(-)$ or vice versa.

Under this model, as in a real grating, the delay introduced by the grating in a given direction cannot be exactly compensated by the delay introduced in the other one.

The phase transfer functions for the cases (+) and (-) are, respectively,

$$
\begin{aligned}
\theta_{+}(\omega)= & \int \tau_{+}(\omega) \mathrm{d} \omega \\
= & c+a_{2} \omega+\frac{a_{1}}{2} \omega^{2} \\
& +b\left(p_{0} \Omega_{F}\right)^{1 / 2} \bar{C}\left[\frac{2 \omega-\Omega_{F}}{\left(p_{0} \Omega_{F}\right)^{1 / 2}}\right] \sin \left(\frac{\pi \Omega_{F}}{2 p_{0}}\right) \\
& -b\left(p_{0} \Omega_{F}\right)^{1 / 2} \bar{S}\left[\frac{2 \omega-\Omega_{F}}{\left(p_{0} \Omega_{F}\right)^{1 / 2}}\right] \cos \left(\frac{\pi \Omega_{F}}{2 p_{0}}\right), \\
\theta_{-}(\omega)= & \int \tau_{-}(\omega) \mathrm{d} \omega \\
= & c+a_{2} \omega+\frac{a_{1}}{2} \omega^{2} \\
& -b\left(p_{0} \Omega_{F}\right)^{1 / 2} \bar{C}\left[\frac{2 \omega+\Omega_{F}}{\left(p_{0} \Omega_{F}\right)^{1 / 2}}\right] \sin \left(\frac{\pi \Omega_{F}}{2 p_{0}}\right) \\
& +b\left(p_{0} \Omega_{F}\right)^{1 / 2} \bar{S}\left[\frac{2 \omega+\Omega_{F}}{\left(p_{0} \Omega_{F}\right)^{1 / 2}}\right] \cos \left(\frac{\pi \Omega_{F}}{2 p_{0}}\right),
\end{aligned}
$$

where $\bar{S}$ and $\bar{C}$ are the Fresnel integrals of sine and cosine respectively:

$\bar{S}(x)=\left(\frac{\pi}{2}\right)^{1 / 2} \int_{0}^{x} \sin t^{2} \mathrm{~d} t, \quad \bar{C}(x)=\left(\frac{\pi}{2}\right)^{1 / 2} \int_{0}^{x} \cos t^{2} \mathrm{~d} t$.

This phase is asymmetric and leads to a net displacement of the frequency of the filtered signal.

If we expand these phase functions in Taylor series around $\omega_{0}$, we obtain, for small displacements,

$$
\theta_{+}(\omega) \approx \theta_{0}+a_{2} \omega+\frac{1}{2}\left(a_{1}-\frac{2 \pi b}{p_{0}}\right) \omega^{2}-\frac{2 \pi b}{3 p_{0} \Omega_{F}} \omega^{3},
$$




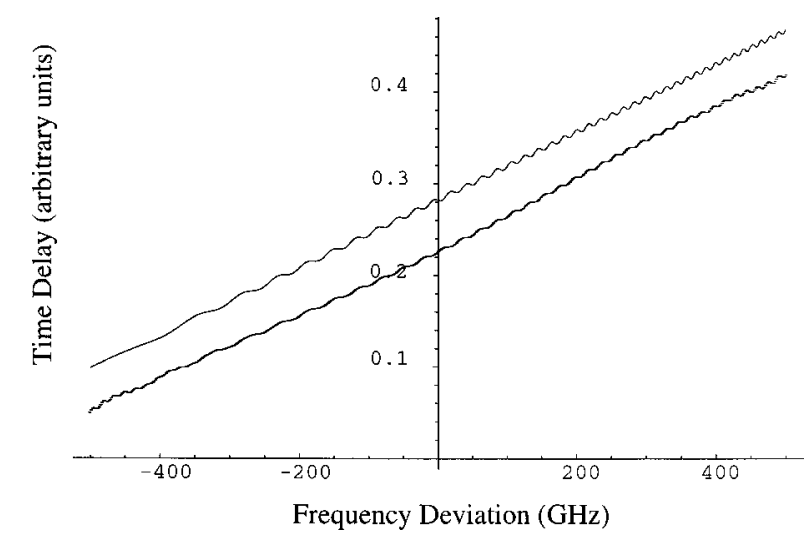

Fig. 5. Time delay of a FBG under the approximation of period variation for the ripples (thin curve), and numerically obtained from the coupled mode equations (thick curve). The simulated time delay is vertically displaced for comparison.

$$
\theta_{-}(\omega) \approx \theta_{0}+a_{2} \omega+\frac{1}{2}\left(a_{1}-\frac{2 \pi b}{p_{0}}\right) \omega^{2}+\frac{2 \pi b}{3 p_{0} \Omega_{F}} \omega^{3},
$$

where $\theta_{0}$ groups all the constant terms,

$$
\begin{aligned}
\theta_{0}= & c+b \frac{\left(p_{0} \Omega_{F}\right)^{1 / 2}}{2}\left[\cos \left(\frac{\pi \Omega_{F}}{2 p_{0}}\right) \bar{S}\left(\frac{\Omega_{F}}{p_{0}}\right)^{1 / 2}\right. \\
& \left.-\sin \left(\frac{\pi \Omega_{F}}{2 p_{0}}\right) \bar{C}\left(\frac{\Omega_{F}}{p_{0}}\right)^{1 / 2}\right] .
\end{aligned}
$$

From this expression we can extract the term corresponding to the third-order dispersion, $C_{3}(\omega)_{ \pm}$,

$$
C_{3}(\omega)_{ \pm}=\mp \frac{2 \pi b}{3 p_{0} \Omega_{F}} \omega^{3} .
$$

This term can be expressed in the usual notation for the third-order dispersion in a segment of fiber, if we take into account that

$$
C_{3}(\omega)_{ \pm}=\frac{\beta_{3_{ \pm}}^{\prime}}{6} L_{\mathrm{g}} \omega^{3}
$$

where $L_{\mathrm{g}}$ is the grating length. Thus

$$
\beta_{3_{ \pm}}^{\prime}=\mp \frac{4 \pi b}{p_{0} \Omega_{F} L_{\mathrm{g}}}
$$

will be the third-order dispersion of the grating, considered as a segment of dispersive fiber, in the proximities of the central frequency. The sign of the third-order dispersion around the central frequency is negative if the period of the ripples increases with $\omega$, and positive otherwise. This term grows with the amplitude of the oscillations. A strong apodization reduces the amplitude of the ripples, $b$, and thus every third- or higher-order dispersion term in the chirped FBG.

The mechanism that leads to the formation of the multisoliton bound states seems to depend critically on the presence of higher-order dispersive terms in the gratingtransfer function, which are responsible for the signal assymetrization. ${ }^{21}$ Thus third-order dispersion in the fiber link should have a high influence on the formation of the multisolitons. The sign of the dispersive term introduced by our gratings at the central frequency of the signal is, following the criterion presented in the previous section, positive. It is responsible, together with the nonlinear effects, for a net displacement of the central frequency of the signal to higher frequencies.

Typical values for $\beta_{3}$ on dispersion-shifted fibers ${ }^{26}$ are $\sim 0.06 \mathrm{ps}^{3} / \mathrm{km}$. We have studied the formation of bound states under different values of $\beta_{3}$, both negative and positive. Values of $\beta_{3}$ between -0.04 and +0.05 do not prevent the formation of the multisoliton state, while values higher than 0.05 and lower than -0.04 break the necessary equilibrium for the formation of the bound state, though they act in a different manner. A high positive $\beta_{3}$ leads to the collapse of a pair of pulses after a long propagation distance (in general, longer than $30 \mathrm{Mm}$ ), reducing the distance between them to zero. On the other hand, a highly negative $\beta_{3}$ is responsible for a gradual increase of the pulse separation that eventually prevents the formation of the bound state due to the lack of interaction.

In Fig. 6 we show the evolution in the interaction plane of a pair of solitons for $\beta_{3}=0.063 \mathrm{ps}^{3} / \mathrm{km}^{-1}$ and $\beta_{3}$ $=-0.063 \mathrm{ps}^{3} / \mathrm{km}^{-1}$ for a propagation distance of $60 \mathrm{Mm}$. This distance is not reached in the case of positive propagation, due to the previous collapse of the signal.

Positive values of $\beta_{3}$ under $0.02 \mathrm{ps}^{3} / \mathrm{km}^{-1}$ reduce the propagation distance necessary for the formation of a bound state, so we can deduce that the third-order dispersion introduced by the gratings is slightly lower than that most suitable for the formation of the bisoliton state. By varying the third-order dispersion of a system initially not suitable for the formation of multisoliton states, we

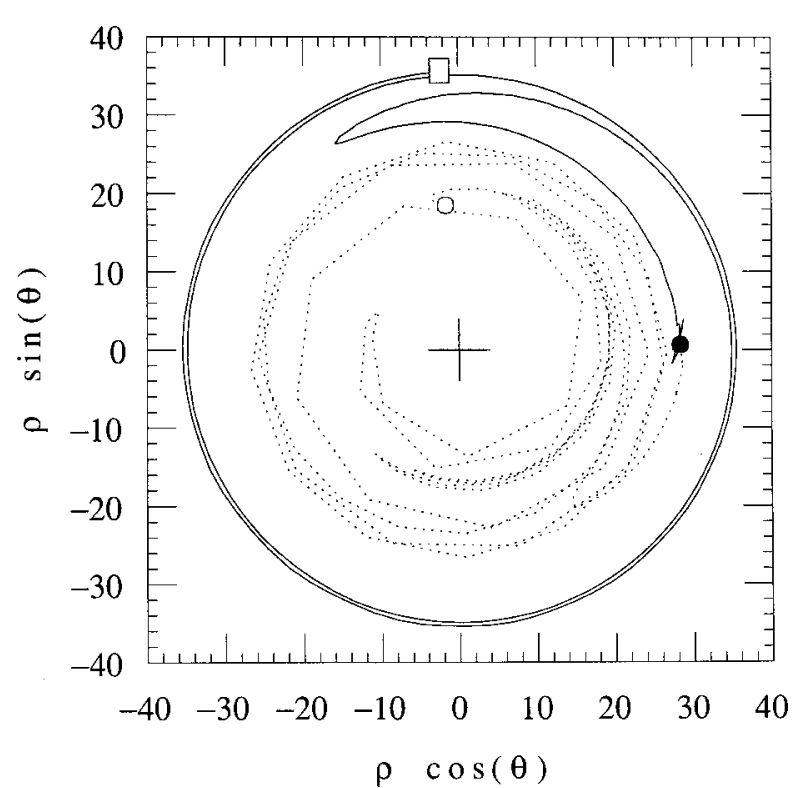

Fig. 6. Evolution in the interaction plane of a pulse pair, initially identical and in phase, with $\beta_{3}=-0.063 \mathrm{ps}^{3} / \mathrm{km}$ (continuous curve) and $\beta_{3}=0.063 \mathrm{ps}^{3} / \mathrm{km}$ (dotted curve). The common origin of both trajectories is marked in the figure with a black dot. The open square signals the situation for the $\beta_{3}<0$ case at $60 \mathrm{Mm}$, while the open circle shows the situation for the case $\beta_{3}>0$ at $36.4 \mathrm{Mm}$, just before the collapse of the signal. $\rho$ and $\theta$ are, respectively, the time distance and the phase difference between peaks. 
can make the system adequate to bear such states, or even accelerate the formation of bound states in a system that can initially bear them.

\section{EFFECTS OF RANDOM VARIATION}

Random variation of the FBGs' parameters is introduced to simulate the experimental differences between gratings in the fiber link. First, we analyze variation of the parameters alone, and then we will study the combined effect of this variation with the presence of third-order dispersion in the fiber link.

The system presents different tolerance to the variation of each of the three parameters, but is, in general, robust to such variations. Though it may seem surprising, small oscillations of the parameters are beneficial for the formation of the bound state and accelerate the bounding process. This is typical of some systems near the equilibrium, because the oscillations help soliton pairs avoid local stability points on the way toward the absolute minimum of potential. Table 2 summarizes the different ranges of beneficial (B), negative (N), and destructive (D) effects variation of the parameters has on the formation of the bound state. We consider the variation beneficial if it accelerates the formation of the bound state, negative if it produces a delay of the formation, and destructive if it prevents the formation of the bound state. These ranges are extremely dependent on the mean grating, so only general information on the tendencies can be obtained from the table. The table has been created taking into account the variation of each of the parameters separately. A simultaneous variation can still produce a beneficial result only if it is not much greater than $1 \%$, but produces a highly negative or deleterious result if greater. These ranges can be modified by any change in the system's operating conditions, such as the presence of thirdorder dispersion in the fiber link.

Table 2 shows that the system is more sensitive to length variations. This is not surprising, because the grating-transfer functions are also more sensitive to length than to any other parameter. Relatively large variations of the coupling parameter $\kappa$ lead to an instability in the distance between pulses, while similar variations on the chirp parameter $F$ produce an instability in the relative phases. Length variations affect the convergence in a global manner, contributing to instabilities both in the relative phase and in the distance between

Table 2. Robustness Against Grating Parameter Variations $^{a}$

\begin{tabular}{cccc}
\hline Variation & $L$ & $\kappa$ & $F$ \\
\hline 0.01 & $\mathrm{~B}$ & $\mathrm{~B}$ & $\mathrm{~B}$ \\
0.02 & $\mathrm{~N}$ & $\mathrm{~B}$ & $\mathrm{~N}$ \\
0.03 & $\mathrm{~N}$ & $\mathrm{~N}$ & $\mathrm{~N}$ \\
0.04 & $\mathrm{~N}$ & $\mathrm{~N}$ & $\mathrm{~N}$ \\
0.05 & $\mathrm{D}$ & $\mathrm{N}$ & $\mathrm{N}$ \\
0.06 & $\mathrm{D}$ & $\mathrm{D}$ & $\mathrm{D}$ \\
0.07 & $\mathrm{D}$ & $\mathrm{D}$ & $\mathrm{D}$ \\
\hline
\end{tabular}

${ }^{a} \mathrm{~B}$, beneficial influence; $\mathrm{N}$, negative influence; and $\mathrm{D}$, destructive influence.

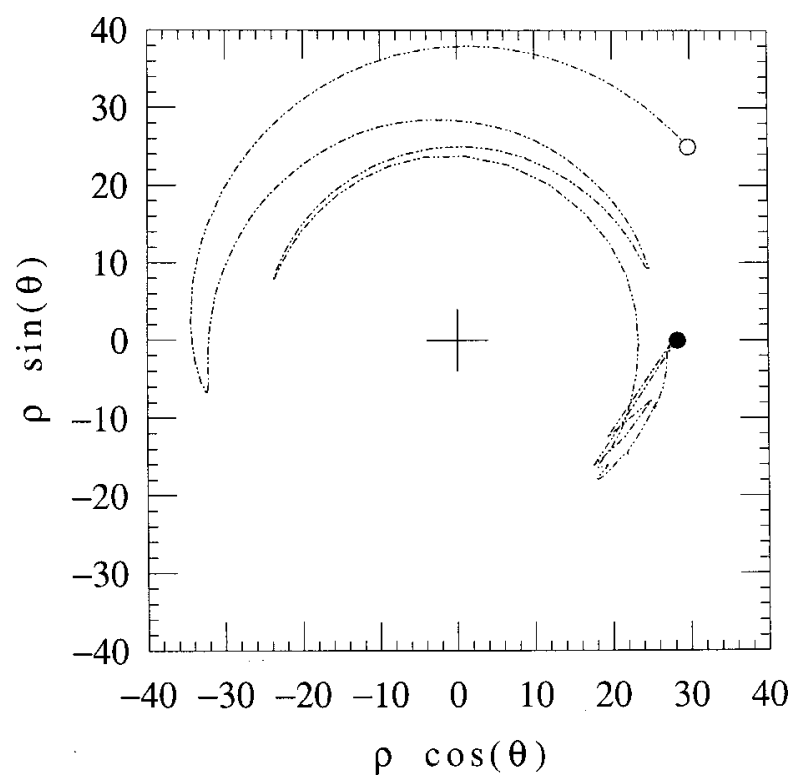

Fig. 7. Evolution of a soliton pair in the interaction plane through $60 \mathrm{Mm}$ with a random variation of the grating length of up to $5 \%$. The black dot represents the origin, while the open circle represents the situation at a distance of $60 \mathrm{Mm}$.

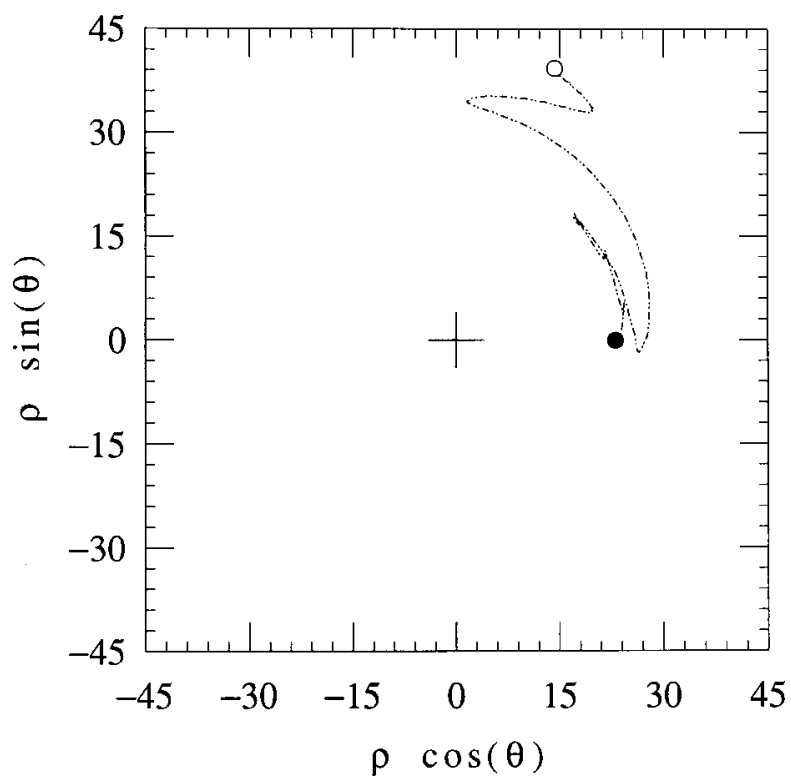

Fig. 8. Same as Fig. 7, but with a random variation of the grating coupling constant $\kappa$ of up to $5 \%$.

pulses. Figures 7, 8, and 9 show the evolution in the interaction plane for a pair of pulses with independent variations of $5 \%$ for each parameter. In none of the three cases does the initial pulse pair converge to a stable solution. This happens independently of the initial separation between pulses.

The most realistic case for our system, in which we consider a typical third-order dispersion in the fiber link and slight variations of the grating parameters, is appropriate for the formation of bound states. Though, as mentioned above, values of $\beta_{3}$ near $0.06 \mathrm{ps}^{3} / \mathrm{km}^{-1}$ prevent the formation of the bound state if no variation of the parameters is considered, the inclusion of such variations partially counteracts the effect of third-order dispersion, making the convergence possible. 


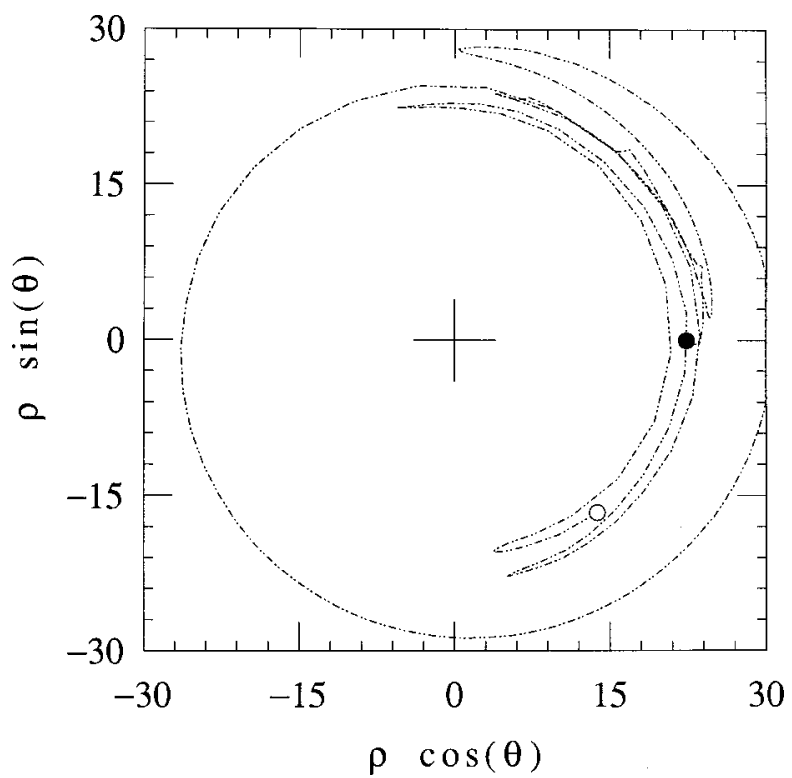

Fig. 9. Same as Fig. 7, but with a random variation of the grating chirp parameter $F$ of up to $5 \%$.

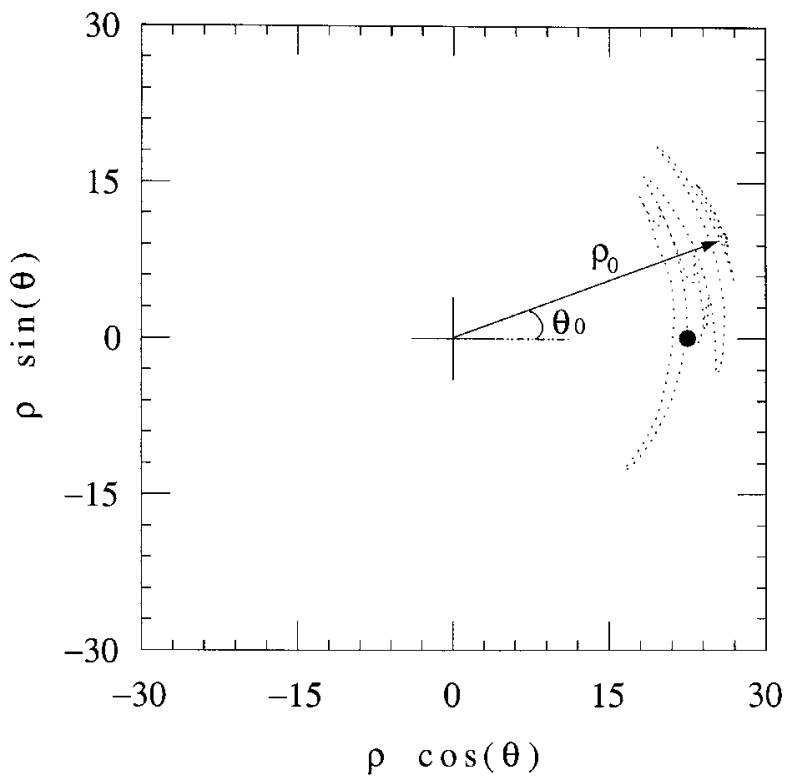

Fig. 10. Evolution in the interaction plane of a pulse pair through $60 \mathrm{Mm}$ for the case $\beta_{3}=0.063 \mathrm{ps}^{3} / \mathrm{km}^{-1}$ with a random variation of $1 \%$ for $L$ and $F$ and of $1.5 \%$ for $\kappa$. The origin is represented with a black dot, $\rho_{0}$ and $\theta_{0}$ are the coordinates of the bound state.

Figure 10 shows the evolution on the interaction plane of a pair of pulses for the case $\beta_{3}=0.063 \mathrm{ps}^{3} / \mathrm{km}^{-1}$, with a random variation of the parameters of $1 \%$ for $L$ and $F$, and $1.5 \%$ for $\kappa$.

The variation of the coupling parameter $\kappa$ is slightly out of the beneficial range stated for a simultaneous variation of the three parameters in the absence of thirdorder dispersion in the link. In this case, due to the presence of third-order dispersion, pulses tend to collapse, and the instability in the distance between pulses caused by the variation of the coupling becomes more useful than it was in the absence of third-order dispersion, thus helping to avoid the destruction of the signal.

\section{CONCLUSIONS}

Multisoliton pulses are naturally generated in nonideal fiber Bragg grating dispersion-managed systems due to the presence of asymmetric terms in the transfer function of the gratings. The inclusion of a full grating model is necessary to explain the effects that lead to the formation of bound states. The shape of these states, with a fixed distance between peaks, sets a limit to the bit capacity of the channel at distances of several megameters. The distance between peaks in the bound state is shorter than the distance between traditional or dispersion-managed neighboring interacting solitons. Because two solitons initially set at twice the peak distance of the bound state do not interact in an appreciable manner, these bound states can be used to increase the capacity of the channel. The formation of bound states depends on the mean thirdorder dispersion introduced by the different devices through the propagation. This property should be considered in any possible design or modification of a given system with the intention of using the new bound states. High values for the third-order dispersion in the fiber link, both positive or negative, can prevent the formation of the bound state.

The differences between the transfer functions of supposedly identical fiber Bragg gratings employed in the formation of multisoliton states can be beneficial for the formation of such states. In our system, this happens if the simultaneous variations of all the parameters are not higher than $1 \%$, though the system is more sensitive to length variations than to changes in the coupling parameter $\kappa$ or the chirp parameter $F$. The ranges of beneficial influence of the parameters' variation are dependent on the system conditions. Variations greater than $1 \%$ of the gratings' parameters can hamper or prevent the formation of bound states.

The formation of a bound state under realistic values for the third-order dispersion in the fiber link is possible in combination with slight random variations of the gratings' parameters.

\section{ACKNOWLEDGMENTS}

J. D. Ania-Castañón wishes to thank the Comunidad de Madrid for the financial support of his work. This work has been supported by the Comisión Interministerial de Ciencia y Tecnología, Spain, Projects TIC 99-0645-c05-03 and PB-96-0819.

\section{REFERENCES}

1. N. J. Smith, N. J. Doran, W. Forysiak, and F. M. Knox, "Soliton transmission using periodic dispersion compensation," J. Lightwave Technol. 15, 1808-1822 (1997).

2. J. P. Gordon and L. F. Mollenauer, "Scheme for the characterization of dispersion-managed solitons," Opt. Lett. 24, 223-225 (1999).

3. D. S. Govan, W. Forysiak, and N. J. Doran, "Long-distance 40-Gbits soliton transmission over standard fiber by use of dispersion management," Opt. Lett. 23, 1523-1525 (1998).

4. M. Nakazawa and H. Kubota, "Optical soliton communication in a positively and negatively dispersion-allocated optical fiber transmission line,” Electron. Lett. 31, 216-217 (1995). 
5. C. Paré and P. A. Bélanger, "Antisymmetric soliton in a dispersion-managed system," Opt. Commun. 168, 103-109 (1999).

6. J. H. B. Nijhof, W. Forysiak, and N. J. Doran, "Dispersionmanaged solitons in the normal dispersion regime: A physical interpretation," Opt. Lett. 23, 1674-1676 (1998).

7. G. M. Carter, J. M. Jacob, C. R. Menyuk, E. A. Golovchenko, and A. N. Pilipetskii, "Timing-jitter reduction for a dispersion-managed soliton system: Experimental evidence," Opt. Lett. 22, 513-515 (1997).

8. T. I. Lakoba and R. S. Tasgal, "Novel mechanism of suppression of radiation by dispersion-managed solitons in randomly birefringent fibers," Technical Digest of Conference on Lasers and Electro-Optics (Optical Society of America, Washington, D.C., 2000), paper CMF6, p. 31.

9. S. Kumar and A. Hasegawa, "Quasi-soliton propagation in dispersion-managed optical fibers," Opt. Lett. 22, 372-374 (1997).

10. Y. Kodama, S. Kumar, and A. Maruta, "Chirped nonlinear pulse propagation in a dispersion-compensated system," Opt. Lett. 22, 1689-1671 (1997).

11. S. K. Turytsin and V. M. Mezentsev, "Chirped solitons with strong confinement in transmission links with in-line fiber Bragg gratings," Opt. Lett. 23, 600-602 (1998).

12. J. D. Ania-Castañón, P. García-Fernández, and J. M. SotoCrespo, "Stable multisoliton pulses in dispersion management with fiber Bragg gratings," Opt. Lett. 25, 159-161 (2000).

13. Y. Chen and H. A. Haus, "Dispersion-managed solitons in the net positive dispersion regime," J. Opt. Soc. Am. B 16, 24-30 (1999).

14. K. O. Hill and G. Meltz, "Fiber Bragg grating technology fundamentals and overview," IEEE J. Lightwave Technol. 15, 1263-1276 (1997).

15. S. H. Yun, D. J. Richardson, and B. Y. Kim, "Interrogation of fiber grating sensor arrays with a wavelength-swept fiber laser," Opt. Lett. 23, 843-845 (1998).

16. G. P. Agrawal, Nonlinear Fiber Optics (Academic, San Diego, Calif., 1989).

17. F. Oullette, "Dispersion cancellation using linearly chirped Bragg grating filters in optical waveguides," Opt. Lett. 12, 847-849 (1987).

18. V. V. Afanasjev and N. N. Akhmediev, "Soliton interaction in nonequilibrium dynamical systems," Phys. Rev. E 53, 64716475 (1996).

19. N. N. Akmediev, A. Ankiewicz, and J. M. Soto-Crespo, "Multisoliton solutions of the complex Ginzburg-Landau equation," Phys. Rev. Lett. 79, 4047-4051 (1997).

20. J. M. Soto-Crespo and N. N. Akhmediev, "Multisoliton regime of pulse generation by lasers passively mode locked with a slow saturable absorber," J. Opt. Soc. Am. B 16, 674677 (1999).

21. T. I. Lakoba and G. P. Agrawal, "Effects of third-order dispersion on dispersion-managed solitons," J. Opt. Soc. Am. B 16, 1332-1343 (1999).

22. A. Hasegawa, Y. Kodama, and Y. Kodama, Solitons in Optical Communications, Vol. 7 of Oxford Series in Optical and Imaging Science (Clarendon, Oxford, 1995).

23. R. Kashyap and M. de Lacerda Rocha, "On the group delay characteristics of chirped fiber Bragg gratings," Opt. Commun. 153, 19-22 (1998).

24. R. Kashyap, Fiber Bragg Gratings, Optics and Photonics Series (Academic, San Diego, Calif., 1999).

25. K. Ennser, M. Ibsen, M. Durkin, M. N. Zervas, and R. I. Laming, "Influence of nonideal chirped fiber Bragg grating characteristics on dispersion cancellation," IEEE Photon. Technol. Lett. 10, 1476-1478 (1998).

26. E. Iannone, F. Matera, A. Mecozzi, and M. Settembre, Nonlinear Optical Communication Networks (Wiley, New York, 1998). 